\title{
Large-Angle Electron Diffraction Structure in Laser-Induced Rescattering from Rare Gases
}

\author{
D. Ray, ${ }_{1}^{1}$ B. Ulrich, ${ }^{2}$ I. Bocharova, ${ }^{1}$ C. Maharjan, ${ }^{1}$ P. Ranitovic, ${ }^{1}$ B. Gramkow, ${ }^{1}$ M. Magrakvelidze, ${ }^{1}$ S. De,${ }^{1}$ \\ I. V. Litvinyuk, ${ }^{1}$ A. T. Le, ${ }^{1}$ T. Morishita, ${ }^{1,3}$ C. D. Lin, ${ }^{1}$ G. G. Paulus, ${ }^{4}$ and C. L. Cocke ${ }^{1}$ \\ ${ }^{1}$ J. R. Macdonald Laboratory, Physics Department, Kansas State University, Manhattan, Kansas 66506-2601, USA \\ ${ }^{2}$ Institut für Kernphysik, University Frankfurt, Max von Laue Str. 1, D-60438 Frankfurt, Germany \\ ${ }^{3}$ Department of Applied Physics and Chemistry, The University of Electro-Communications, \\ 1-5-1 Chofu-ga-oka, Chofu-shi, Tokyo 182-8585, Japan \\ and PRESTO, Japan Science and Technology Agency, Kawaguchi, Saitama 332-0012, Japan \\ ${ }^{4}$ Institute of Optics and Quantum Electronics, 07743, Jena, Germany \\ and Department of Physics, Texas A\&M University, College Station, Texas 77843-4242, USA
}

(Received 18 December 2007; published 9 April 2008)

\begin{abstract}
We have measured full momentum images of electrons rescattered from $\mathrm{Xe}, \mathrm{Kr}$, and Ar following the liberation of the electrons from these atoms by short, intense laser pulses. At high momenta the spectra show angular structure (diffraction) which is very target dependent and in good agreement with calculated differential cross sections for the scattering of free electrons from the corresponding ionic cores.
\end{abstract}

PACS numbers: $32.80 . \mathrm{Rm}, 32.80 . \mathrm{Wr}, 34.50 .-\mathrm{s}$

When a short, intense laser pulse is applied to an atom one (or more) electron(s) can be removed. The electron does not leave the vicinity of the parent ion but is driven back to the ionic core by the laser where it may then undergo a variety of "rescattering" processes, including high harmonic generation [1], inelastic excitation and ionization of the core [2,3], and elastic scattering (diffraction) [4-6]. Because the effective current density of the returning electrons [3] is much higher than that which could be realized by sending free electrons onto the ions in the laboratory, many efforts are underway to bring under control the use of the returning electron flux to image the parent atom or molecule, ultimately in a time-resolved manner. A central question to this effort is: to what extent can the properties of the returning wave packet be separated from the characteristics of the differential scattering? Observing the latter is the major goal in the quest for imaging. Morishita et al. [7] have recently calculated that the clean separation of the characteristics of the returning wave packet and those of the free-electron differential cross section is possible. They pointed out that differential scattering cross sections for low energy electrons from rare gas (and other) ions at low energies are well-known $[7,8]$ to typically produce marked angular structure in the backwards hemisphere which is sensitive to the specific ion. They showed that full solutions to the time-dependent Schrödinger equation including rescattering allow the identification of specific "back-rescattering ridges" (BRR) along which the angular structure of the differential cross section is clearly visible and very target dependent. In this Letter we report experimental observation of these predicted features.

Electron spectra from intense-laser-induced ionization of atoms are well-known to be characterized by two components [9]. "Direct" electrons are liberated from the host approximately at rest and are subsequently acted upon by the laser field to result in final energies up to $2 U_{p}$ (where
$U_{p}$ is the oscillation energy of a free electron in the laser field), the particular energy depending on the phase of the field at which they are released. "Plateau" electrons with energies up to $10 U_{p}$ are generated by the rescattering of returning direct electrons. The periodic nature of the laser field results in an above-threshold-ionization (ATI) structure superimposed on the electron spectrum with peaks separated by a single photon energy. To some extent the diffraction effects reported and interpreted here were anticipated by studies more than a decade ago. Yang et al. [10] first reported the result that the angular widths of the ATI spectra showed a broadening near $9 U_{p}$ and suggested that this had to do with the characteristics of the rescattering cross section from the ionic core. Similar observations and theoretical analysis followed [5,6,11-19]. It was also known that the atomic structure of the target must play an important role in the exact nature of the backscattering from the observation that the electron spectra for different gases (for example, $\mathrm{Xe}, \mathrm{Kr}$, and $\mathrm{Ar}$, the gases used here) are quite different [5]; similar marked differences have been seen for K and Na. [19]. The key role played by the marked differential backscattering cross section was previously suspected [10,20] but not previously quantitatively examined.

The BRR predicted by Morishita et al. lie near $10 U_{p}$ and represent the highest energy backscattering electrons at the very edge of the ATI plateau. In a classical interpretation, these electrons result from electrons which were ionized from the target near a phase of $17^{\circ}$ beyond the peak field, returning with an energy near $3 U_{p}$, or a momentum $p_{0}=$ $1.22 A_{0}$, where $A_{0}$ is the peak value of the vector potential and both $p_{0}$ and $A_{0}$ are in atomic units (a.u.). At the time of recollision the vector potential is near $0.95 A_{0}$ [7]; this is the additional momentum which the rescattered electrons will gain from the field after the collision. The locus of these events in momentum space, shown in Fig. 1(a), is thus a 

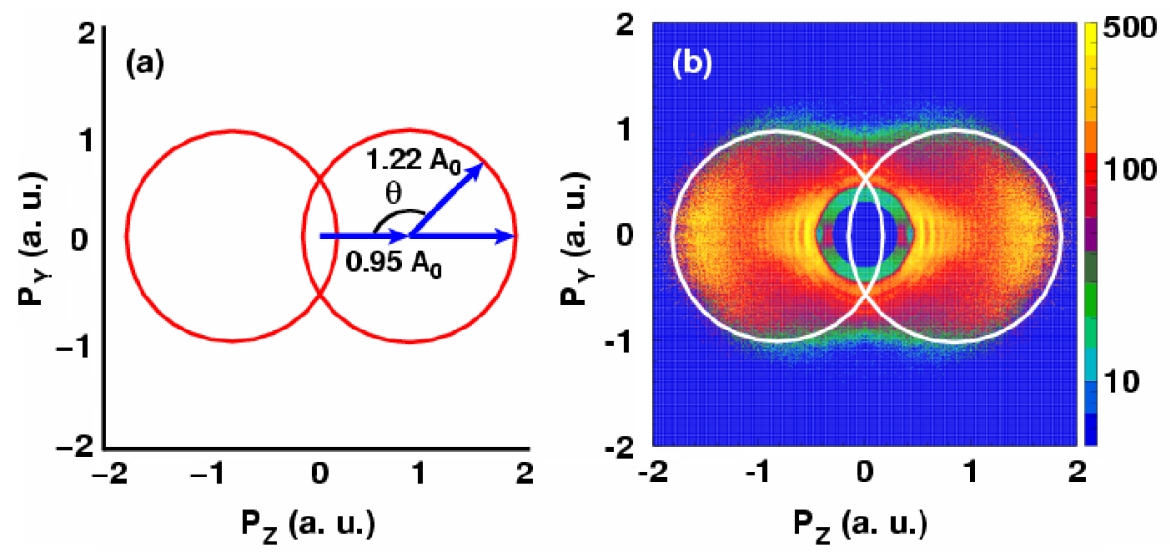

FIG. 1 (color online). (a) Schematic showing the relative sizes of the momentum vector of a returning electron with maximal energy and the momentum shift which this electron will receive from the laser field after recollision, at $8.3 \times$ $10^{13} \mathrm{~W} / \mathrm{cm}^{2}$. (b) Experimental electron momentum image from a 7 fs pulse of this intensity on the background gas of water vapor and hydrocarbons. pair of circular ridges, the BRR, centered at $p_{z}= \pm 0.95 A_{0}$ and with radii of $1.22 A_{0}$, where we take the laser polarization to be along the $z$ axis and the momentum vector of the electron to lie in the $y-z$ plane.

We have used experimental parameters which focus on a reliable measurement, with good statistics, of the high energy end of the plateau, using a modified version of the time-honored time-of-flight method. A 7 fs pulse with a central wavelength near $800 \mathrm{~nm}$ was generated by passing a 35 fs pulse through a Ne-gas-filled fiber, followed by chirped mirrors and compensating glass. The pulse length was determined using frequency resolved optical gating. The pulses were focused to an intensity between 4 and $9 \times$ $10^{13}$ watts $/ \mathrm{cm}^{2}$ into a small gas cell consisting of a $2 \mathrm{~mm}$ canal $2 \mathrm{~mm}$ long and containing the target gas at $0.2-$ 2 mTorr. The electrons emerged transversely from two small $0.5 \mathrm{~mm}$ apertures in a field free spectrometer, traveling $15.7 \mathrm{~cm}$ in both left and right directions (the $x$ direction) to $2.5-\mathrm{cm}$-diameter channel plate detectors, each therefore subtending a maximum angular range of $\pm 4.6^{\circ}$. Individual electron pulses were discriminated with constant fraction discriminators (CFD) and fed to a multihit time-to-amplitude converter. The momenta were calculated from the flight times on an electron-by-electron basis. The angle between the electron emission and the polarization vector was varied using an achromatic half-wave plate. The target cell gas pressure was typically adjusted in such a way that, on the average, one electron per pulse was detected in the region of $10 U_{p}$. Under these conditions the momentum of the first hit is accurately recorded, but the detection efficiency for lower energy electrons is substantially reduced, in spite of the use of a multihit time-todigital converter, by the dead time (20 ns) of the CFD.

Figure 1(b) shows a spectrum taken with the cell containing water vapor and hydrocarbons, the background gas in our system (evaluated using a residual gas analyzer). Similar to the procedure in Ref. [7], the image has been normalized to emphasize larger electron energies $\left(E_{e}\right)$, in this case by dividing by the factor $e^{-\left(E_{e} / 10 \mathrm{eV}\right)}$. The outer edges of the BRR are clearly apparent, but the ridges themselves appear more as discs than as isolated ridges.
This is expected, since the experiment volume averages, and thus represents the yield over a range of intensities beginning at the maximum intensity. The outer edge of each disk has an aspect ratio (1.22 radius to 0.95 shift) very close to that expected for the BRR from the classical picture. This aspect ratio for the outer edge is robust in all of our spectra on the background gas. Unlike the result for rare gas targets discussed later, little angular structure along the circles is seen.

Figure 2 shows the corresponding images for a Xe target at four different intensities. Now a clear minimum is observed near a laboratory angle $\theta_{\text {lab }}$ of $150^{\circ}$ or centerof-mass angle of $\theta$ of $140^{\circ}$, with a weaker minimum near $100^{\circ}$, as expected from theory. Here $\theta$ is measured with respect to the shifted origin of the BRR, as shown in
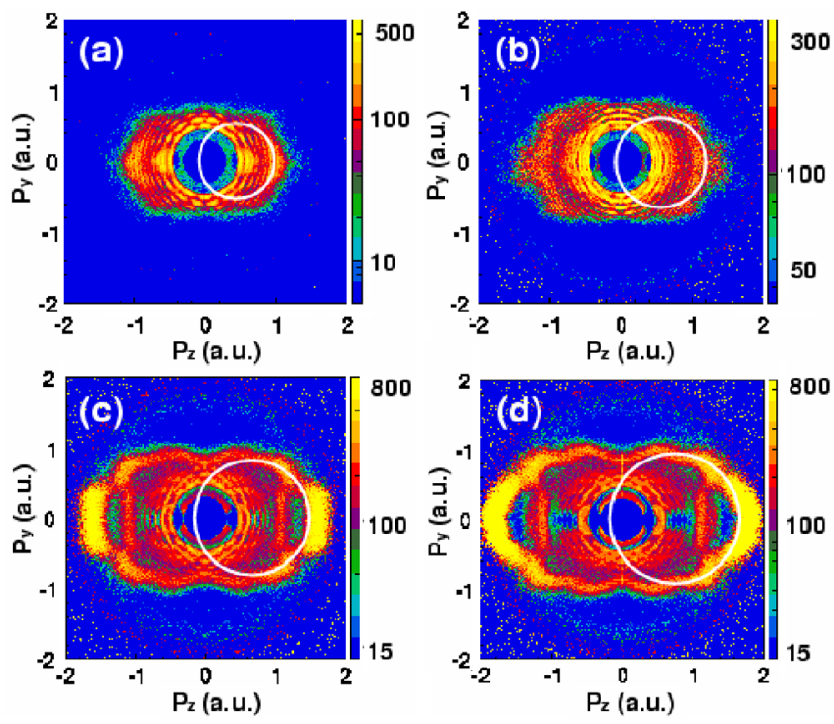

FIG. 2 (color online). Density plots of electron momentum spectra at different laser intensities for $7 \mathrm{fs}$ pulses on Xe. The intensities (in units of $10^{13} \mathrm{~W} / \mathrm{cm}^{2}$ ) and corresponding values of $p_{0}$ (in a.u.) are, respectively, (a) 4.18, 0.74; (b) 5.8, 0.86; (c) 7.1, 0.95 ; (d) $8.3,1.03$. The white circles show the inner edges of the regions from which the differential cross sections were extracted (see text). 
Fig. 1(a), and represents the angle through which the electron is rescattered. The minima are clearly visible in the oscillatory behavior of the outer edge of the images. As expected, the whole momentum picture expands as the intensity, or $A_{0}$, is raised, but the angular structure remains. We note that the apparent ridges [rather than the discs of Fig. 1(b)] seen in some of these spectra are partially caused by the CFD dead time effect discussed above.

We have extracted approximate differential cross sections for electron scattering along the BRR as follows. For each laser intensity we obtained the center of the semicircular pattern from the background scan. From this displaced center we then plotted, as a function of $\theta$, the yield of events on a circular slice extending to infinity from a momentum radius slightly $(10 \%-15 \%)$ inside the outside edge of the observed data (white rings in Fig. 2). This region approximately represents those events from the BRR corresponding to the maximum laser intensity in the volume. While the results are somewhat dependent on the radii chosen, the major features are not. The extracted angular distributions for Xe are shown as data points in Fig. 3, where strong minima are observed near $\theta=140^{\circ}$ and weaker ones near $100^{\circ}$. The solid lines are calculated for free electron scattering on $\mathrm{Xe}^{+}$at a collision energy of $3 U_{p}$ or collision momentum of $p_{0}$ [7]. We have assigned intensities to our experimental data by requiring that the outermost edge of the observed experimental semicircles in the $z$ direction correspond to $9.5 U_{p}$. The results are consistent with an independent calculation of the intensities based on measured powers and focal spot sizes.

In Fig. 4 we compare spectra and extracted angular distributions for $\mathrm{Xe}, \mathrm{Kr}$, Ar, and the background at the same intensity. It is known that $\mathrm{Kr}$ produces a substantially reduced plateau in this intensity region [5], and our data confirm this behavior. We can now interpret this as due to the weak, nearly structureless backscattering expected for the electron- $\mathrm{Kr}^{+}$system. For $\mathrm{Ar}$ one expects stronger structure than for $\mathrm{Kr}$ and the data show this.

The theoretical elastic differential cross sections for scattering between free electrons and atomic ions were calculated within the single electron model. Each rare gas atom was approximated by a model potential $V(r)=$ $V_{S}(r)-1 / r$, consisting of a short-range potential $V_{S}$ and a Coulomb potential. The parameters in the $V_{S}$ were adjusted so that the binding energies of the ground state and the first few excited states of the model atom were in good agreement with experimental values. The method for obtaining the parameters for the model potentials as well as some tabulated data are given in Ref. [21]. The calculation of the differential scattering cross sections for such a modified Coulomb potential is treated in quantum mechanics textbooks (e.g., [22,23]). The phase shifts from the short-range potential were calculated for 30 partial waves, while the Coulomb amplitude is analytical. The diffraction minimum in the differential cross section is often attributed to the

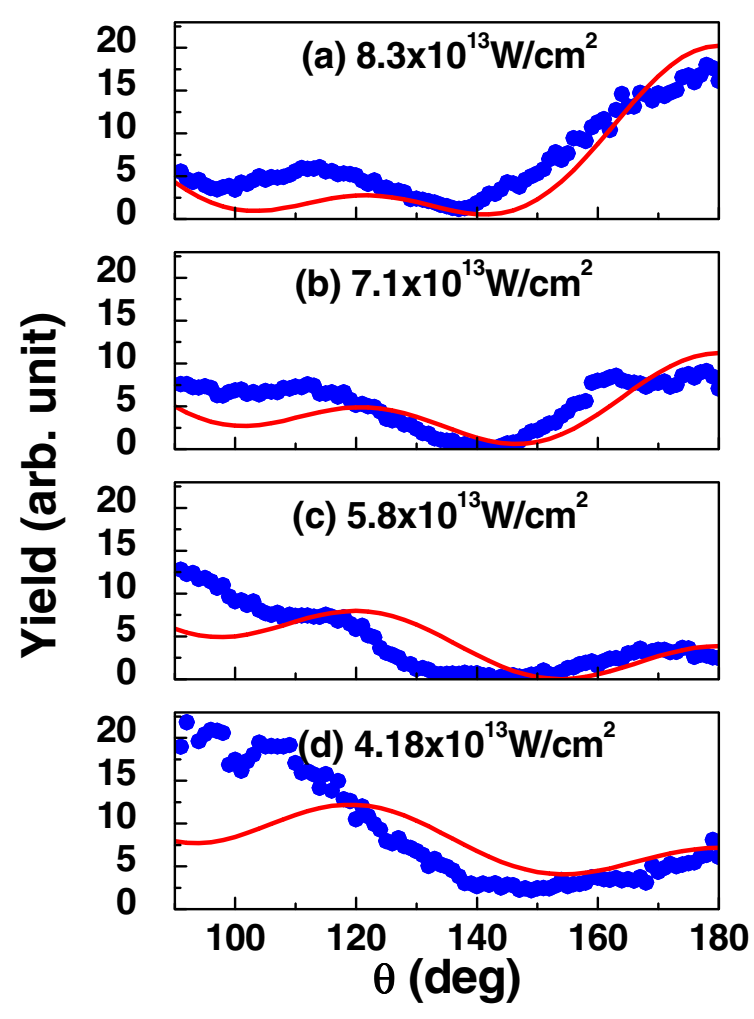

FIG. 3 (color online). Experimental angular distributions extracted from the data of Fig. 2. The solid lines show the theoretical calculations of differential cross sections for free electrons scattered in the backwards direction from $\mathrm{Xe}^{+}$. The momenta of the backscattering electrons, $p_{0}$, are, from top to bottom, 1.03, 0.95, 0.86, and 0.74 a.u. The vertical scale is the theoretical one in atomic units, and the data have been normalized to roughly match the theory.

interference between the scattering amplitudes from the Coulomb potential and the short-range potential. However, this interpretation is not precise since similar minima also occur in the scattering of electrons with neutral atoms.

In conclusion, we have observed strong angular structure in the backscattering of laser-liberated electrons from various targets. For the case of $\mathrm{Xe}$, and to a lesser extent $\mathrm{Ar}$, angular structure along these ridges was observed which can be interpreted as due to the characteristics of the differential cross sections for elastic scattering of free electrons from the corresponding ionic cores. $\mathrm{Kr}$ was found to show only weak structure and a weak backscattering ridge, in agreement with the expected calculation of weak electron backscattering for this target. These observations lend credence to the concept that lasers can be used to produce electron beams in situ, which in turn can be used to diffract from their host targets, and that quantitative information on the structure of the host can be extracted from the observed patterns [24]. For the collision energies addressed here, $7-14 \mathrm{eV}$, the momentum of the electron is too low (de Broglie wavelength too long) to allow the probing of molecular structure. However, if longer wave- 

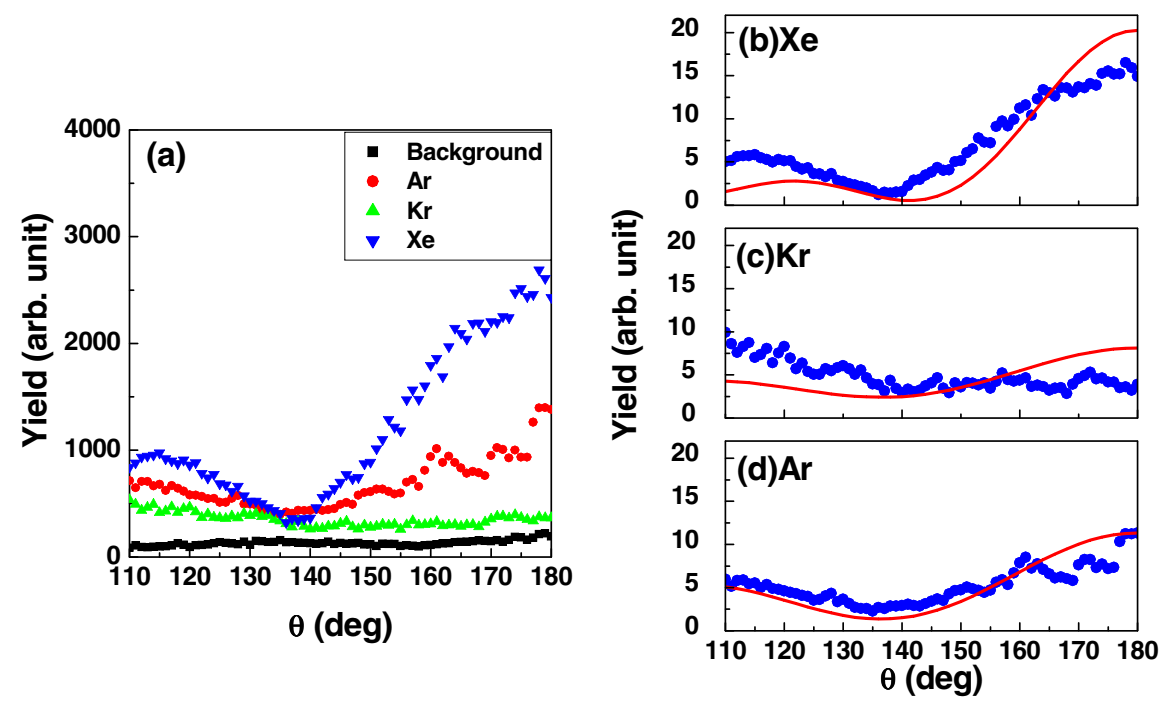

FIG. 4 (color online). (a) Experimental angular distributions for background, Ar, $\mathrm{Kr}$, and $\mathrm{Xe}$ at $8.3 \times 10^{13} \mathrm{~W} / \mathrm{cm}^{2}$. (b)(d) Background-subtracted and normalized angular distributions, compared to theoretical differential cross sections (in a.u.) for backscattering of $14.4 \mathrm{eV}$ free electrons ( $p_{0}=1.03$ a.u. $)$.

lengths are used much higher electron momenta can be obtained, opening the way to the possibility of extracting real diffraction patterns containing structural information on the host molecules, possibly time dependent.

This work was supported by Chemical Sciences, Geosciences and Biosciences Division, Office of Basic Energy Sciences, Office of Science, U. S. Department of Energy, and by the National Science Foundation and the Welch Foundation (G. G.P.).

[1] A. L'Huillier and Ph. Balcou, Phys. Rev. Lett. 70, 774 (1993); J. J. Macklin, J.D. Kmetec, and C.L. Gordon, Phys. Rev. Lett. 70, 766 (1993); M. Lewenstein et al., Phys. Rev. A 49, 2117 (1994); P. B. Corkum, Phys. Rev. Lett. 71, 1994 (1993).

[2] D. N. Fittinghoff et al., Phys. Rev. Lett. 69, 2642 (1992); K. J. Schafer et al.,ibid. 70, 1599 (1993); B. Sheehy et al., Phys. Rev. A 58, 3942 (1998); G. I. Yudin and M. Y. Ivanov, Phys. Rev. A 63, 033404 (2001).

[3] H. Niikura et al., Nature (London) 417, 917 (2002).

[4] T. Zou et al., Chem. Phys. Lett. 259, 313 (1996); M. Spanner et al., J. Phys. B 37, L243 (2004); S. X. Hu and L. A. Collins, Phys. Rev. Lett. 94, 073004 (2005); M. Lein, J. P. Marangos, and P. L. Knight, Phys. Rev. A 66, 051404 (2002); S. N. Yurchenko et al., Phys. Rev. Lett. 93, 223003 (2004).

[5] G. G. Paulus, W. Nicklich, Huale Xu, P. Lambropoulos, and H. Walther, Phys. Rev. Lett. 72, 2851 (1994).

[6] G. G. Paulus, W. Becker, W. Nicklich, and H. Walther, J. Phys. B 27, L703 (1994).
[7] T. Morishita, A. T. Le, Z. Chen, and C. D. Lin, Phys. Rev. Lett. 100, 013903 (2008).

[8] C. Liao, S. Hagmann, C. P. Bhalla, S. R. Grabbe, C. L. Cocke, and P. Richard, Phys. Rev. A 59, 2773 (1999).

[9] W. Becker et al., Adv. At. Mol. Opt. Phys. 48, 35 (2002).

[10] Baorui Yang et al., Phys. Rev. Lett. 71, 3770 (1993).

[11] G. G. Paulus, W. Nicklich, and H. Walther, Europhys. Lett. 27, 267 (1994).

[12] W. Becker, A. Lohr, and M. Kleber, J. Phys. B 27, L325 (1994).

[13] G. G. Paulus, W. Becker, and H. Walther, Phys. Rev. A 52, 4043 (1995).

[14] M.P. Hertlein, P.H. Bucksbaum, and H.G. Muller, J. Phys. B 30, L197 (1997).

[15] G. G. Paulus et al., Phys. Rev. Lett. 80, 484 (1998).

[16] M. J. Nandor, M. A. Walker, and L.D. Van Woerkom, J. Phys. B 31, 4617 (1998).

[17] J.Z. Kaminski and F. Ehlotzky, Phys. Rev. A 55, 4625 (1997).

[18] Jingtao Zhang et al., J. Phys. B 35, 4809 (2002).

[19] M. B. Gaarde et al., Phys. Rev. Lett. 84, 2822 (2000).

[20] F. Grasbon et al., Phys. Rev. Lett. 91, 173003 (2003).

[21] See Table 1 in X.-M.Tong and C. D. Lin, J. Phys. B, 38, 2593 (2005).

[22] A. Messiah, Quantum Mechanics (North-Holland, Amsterdam, 1958), Vol. I, p. 430.

[23] H. Friedrich, Theoretical Atomic Physics (SpringerVerlag, Berlin, 1990), p. 201.

[24] T. Morishita, A. T. Le, Z. Chen, and C.D. Lin, New J. Phys. 10, 025011 (2008). 